\title{
Understanding the mechanisms of cognitive impairments in developmental coordination disorder
}

\author{
Shining Deng ${ }^{1,2}$, Wei-Guang $\mathrm{Li}^{3,4}$, Jing Ding ${ }^{3,4,5}$, Jinlin $\mathrm{Wu}^{1,2}$, Yuanyuan Zhang ${ }^{1,2}, \mathrm{Fei}_{\mathrm{Li}}{ }^{1,2}$ and Xiaoming Shen ${ }^{1,2}$
}

Developmental coordination disorder (DCD), a neurodevelopmental disability in which a child's motor coordination difficulties significantly interfere with activities of daily life or academic achievement, together with additional symptoms of diseases with childhood sensorimotor impairments, increases the risk of many cognitive problems. This exhibits the dynamic interplay between sensorimotor and cognition systems. However, the brain structures and pathways involved have remained unknown over the past decades. Here, we review developments in recent years that elucidate the neural mechanisms involved in the sensorimotor-cognitive difficulties. First, we briefly address the clinical and epidemiological discoveries in DCD as well as its comorbidities. Subsequently, we group the growing evidence including our findings that support the notion that sensorimotor manipulation indeed affects the cognition development at systematic, circuitry, cellular, and molecular levels. This corresponds to changes in diverse brain regions, synaptic plasticity, and neurotransmitter and receptor activity during development under these effects. Finally, we address the treatment potentials of task-oriented sensorimotor enhancement, as a new therapeutic strategy for cognitive rehabilitation, based on our current understanding of the neurobiology of cognitive-sensorimotor interaction.

D evelopmental coordination disorder (DCD) is characterized as a neurodevelopmental disability in which a child's motor coordination difficulties significantly interfere with activities of daily life or academic achievement (1). Historically, it has been variably termed as follows: clumsy child syndrome, sensory integrative dysfunction, developmental dyspraxia, physical awkwardness, perceptual motor dysfunction, and congenital maladroitness (2). According to the Diagnostic and Statistical Manual, Fourth Edition, Text Revision, children with depressive performance that require motor coordination and consequently interfere academic achievement or activities of daily living, while do not have a general medical condition (i.e., cerebral palsy, hemiplegia, or muscular dystrophy) and meet the criteria for a pervasive developmental disorder, can be recognized as the DCD children (1). Presently, depending on the selection criteria used, prevalence estimates for DCD vary from 1.4 to $19.0 \%$ of school-aged children across countries (2). Clinical studies of children with DCD have reported the gender difference in prevalence, where it shows that the gender ratio for boys to girls ranges from 3:1 to as high as 7:1. Even greater prevalence is reported in boys born in unfavorable prenatal environment such as at very low birth weight or too premature, although the potential mechanisms have not been described explicitly. The prevalence, diagnosis, prognosis, and intervention of DCD have been broadly reviewed in other literatures (2).

In recent studies, the main cause for the symptoms of DCD is believed to be the sensorimotor dysfunction produced by pathology in the central nervous system. These beliefs are backed by both modeling, in which the neurological output of motor control accurately predicts the sensory input, and by clinical data, where motor abnormality in DCD children is frequently associated with sensory disability. These children with DCD typically have difficulties with fine and/or gross motor skills, with poor motor performance that is usually slower, less accurate, and more variable than that of their peers (3-12). In reality, the symptoms of DCD are more than just the lower end of normal variance in motor control as it is described initially (13), as it also combines with significantly impacted motor learning ability, hence confronting the patients with challenge in acquiring typical childhood skills, such as tying shoes or riding a bicycle $(3,14)$.

Furthermore, more recent epidemiological studies report that DCD usually coincides with other developmental disorders-for example, attention-deficit and hyperactivity disorder (ADHD). Up to 50\% DCD children have shown the core symptoms of ADHD, such as attention deficiency, hyperactivity, and impulsivity (15-20). Learning disabilities, especially dyslexia, and specific language impairment are also frequently found in DCD children (21), in which 50\% of patients perform abnormally in several assessments of expressive language. Impairment of verbal and visuospatial memory,

\footnotetext{
The first two authors contributed equally to this work.

'Shanghai Institute of Pediatric Translational Medicine, Developmental and Behavioral Pediatric Department, Shanghai Children's Medical Centre, Shanghai Jiao Tong University School of Medicine, Shanghai, China; ${ }^{2}$ Shanghai Key Laboratory of Children's Environmental Health, Shanghai Jiao Tong University School of Medicine, Shanghai, China; ${ }^{3}$ Department of Anatomy and Embryology, Shanghai Key Laboratory for Tumor Microenvironment and Inflammation, Institute of Medical Sciences, Shanghai Jiao Tong University School of Medicine, Shanghai, China; ${ }^{4}$ Department of Biochemistry and Molecular Cell Biology, Shanghai Key Laboratory for Tumor Microenvironment and Inflammation, Institute of Medical Sciences, Shanghai Jiao Tong University School of Medicine, Shanghai, China; ${ }^{5}$ Department of Chinese Medicine, Institute of Pharmacy, Zhejiang Chinese Medicine University, Hangzhou, China. Correspondence: Fei Li (feili@shsmu.edu.cn)
} 


\section{Mechanisms for DCD cognitive impairments}

both short term and working forms, has been detected in DCD young patients at a high frequency (22), suggesting a larger extent of deficiency in learning and memory associated with DCD. Population-based studies indicate that cognitive impairments are commonly accompanying conditions in DCD, and children with DCD may usually have at least one such disorder. It is worthy to note that longitudinal studies have shown that the cognitive impairments in those DCD children may persist into adolescence and adulthood, which may even extend beyond their motor difficulties. Given the high degree of overlap among these above-mentioned developmental disorders, the common causes shared between sensorimotor dysfunction and cognitive impairments have garnered the increasing interest of developmental neurobiologists as well as clinical pediatricians over the past decades.

Functional neuroimaging, pharmacologic, neuroanatomic, and animal studies are beginning to elucidate that cognitive development cannot be isolated from purely sensorimotor function and high-level thinking. It is being shown that appropriate behavior depends on the integration of sensory input and action response. Moreover, specific brain structures and neurotransmitters have been proven to be involved in both cognitive and sensorimotor systems. Cognitive performance related to behavior planning and executive functioning develops in early period of life, when motor processes, such as movement control and visuomotor coordination, develop rapidly. In consideration of the dynamic sensorimotor and cognitive interaction, here we summarize the recent development in the systematic, circuitry, cellular, and molecular mechanisms in relation to DCD. Grounded in the current understanding on the mechanisms of cognitive impairments, we discuss the possibility of the sensorimotor-based correction of the cognitive impairments in patients with DCD.

\section{BRAIN REGIONS INVOLVED IN THE COGNITIVE IMPAIRMENTS OF DCD}

DCD has long been conceptualized as a form of "minimal brain dysfunction" or "minimal neurological dysfunction," both terms of which are used to describe a collection of symptoms reflecting learning, attention, and motor coordination deficits (23). Since DCD as a sensorimotor disorder accompanied by impaired cognition is not just a consequence of one or more neurological disorders or delayed cognitive development, DCD has been proposed as a variant of atypical brain development (24). The possible involvement of specific brain regions in sensorimotor control and cognition in DCD are discussed below.

\section{Cerebellum}

The cerebellum as the core brain region responsible for motor control and motor learning has been firstly speculated to be implicated in DCD. The functional magnetic resonance imaging studies suggest a significant reduction in a broad network of regions including the cerebellum in DCD patients. During the trail-tracing task, after practicing for $3 \mathrm{~d}$, abnormal cerebellum involvement was shown in those with development of DCD (25).
A potential mechanism linking the cerebellum to motor control and motor learning is complex and controversial. One possible explanation is that children with DCD may have difficulty in making motor skills automatic, a physiological function that involves the normal action of the cerebellum. An alternative explanation based on the internal modeling deficit hypothesis was raised recently, where successful motor control is thought to result from an internal model which accurately predicts the sensory consequences of motor command (26). Theoretical models of motor learning posit that the cerebellum receives an efferent copy of the motor command and compares the predicted movement with the actual movement; if there is a mismatch, the cerebellum sends an error signal as feedback to create a more accurate movement on subsequent occasions (27). It is widely believed that cerebellum is indeed involved in the motor symptoms of DCD, regardless of whether through either automatization deficit or internal model deficit.

Furthermore, cerebellum is also regarded to have a bottom-up influence on cognition-related brain regions, which indicates high-level representations built up by elementary sensorimotor memories. Through computational modeling of a functional interplay between the cerebellum and the hippocampal formation during a goal-oriented navigation task, cerebellar involvement was characterized in higher-level aspects of behavioral control positing procedural as well as declarative components of spatial cognition in the hippocampal spatial memory test; rodent cerebellar deficits significantly impacted the exploration-exploitation balance during spatial navigation by influencing the accuracy of hippocampal spatial codes (28). Thus, we may speculate on the existence of a functional cerebellum-hippocampus circuit for cognition regulation. Meanwhile, it has been shown that aberrant activation of cerebellum is frequently observed in the patients with attention deficit which is characterized as prefrontal cortex (PFC) dysregulation, indicating a potential neuronal linkage between cerebellum and other attention-related brain networks. Hence, it could be reasoned that the disrupted cerebellar modulation might account at least in part for the mechanisms of cognitive impairments in young DCD patients.

\section{Prefrontal Cortex}

As a high frequency of coincidence is found between DCD and ADHD, it is speculated that DCD may share etiology with ADHD through some brain structures and pathways, on which increased attention was attracted to focus. One possible common neurological substrate proposed for the cooccurrence of DCD and ADHD is the PFC, with up to $50 \%$ of children with DCD showing attention deficit that are consistent with ADHD. The PFC is recently regarded as motor-related brain region especially responsible for motor learning. The functional magnetic resonance imaging studies have shown that when performing a fine motor practice task, children with DCD had greater brain activation than controls in the frontal brain areas, consistent with the clinical observations that more efforts were required by DCD children when engaging in motor-based activities (29). Following continuance of the 
practice for $3 \mathrm{~d}$, when the children were subjected to retest, a significant reduction was noted in DCD children in right dorsolateral PFC, further strengthening the association between the poor performance of motor learning and disrupted prefrontal regulation in DCD. Moreover, the lower activation in the dorsolateral PFC in DCD was found when compared with control children during a "go/no-go" task (30).

The PFC is located in the anterior part of the frontal lobes of the brain, divided into dorsal and ventral subregions, just in front of the motor and premotor cortex. In addition to the functional role of motor learning, PFC is well believed to be critical for the cognitive-executive function, which is considered to be orchestration of thoughts and actions in accordance with internal goals $(31,32)$. In humans, executive functions, such as planning and organization, behavioral control and adaptation, and decision making, greatly rely on an intact PFC (33). Thus, the dysfunction found in PFC of DCD children might account for either cognitive impairments or sensorimotor dysfunction. In addition, PFC is highly interconnected with much of the brain regions including other cortical, subcortical, and brain stem sites, pushing information processing among prefrontal thalamus, prefrontal hippocampus, prefrontal striatum, and prefrontal cerebellum (34). Specifically, the dorsal part of PFC is highly interconnected with other brain regions of attention networks (35), indicating the dysfunction of PFC and other brain regions at neuronal circuit level likely responsible for attention deficit occurred in DCD. And the ventral part of PFC extensively interconnects with brain regions involved with emotion (36), probably accounting for the emotional symptoms frequently found in DCD children (37-41). Similarly, learning disabilities and language disorders that are usually cooccurred with DCD may also be due to the involvement of PFC and related circuits.

\section{Striatum}

Striatum, also known as the neostriatum or striate nucleus, is a subcortical part of the forebrain. By functional magnetic resonance imaging, the researchers found that DCD children with motor difficulties displayed reduced path coefficients between striatum and parietal cortex in "go/no-go" task (30), indicating striatum involvement in DCD. Functionally, the striatum is the major input station of the basal ganglia system. It, in turn, gets input from the cerebral cortex (42). Striatum is best known for its role in the planning and modulation of movement pathways but is also potentially involved in a wide variety of cognitive processes including executive function, such as working memory, cognitive flexibility, and habit formation (43).

Here, we propose a possible involvement of the striatum in contributing to both sensorimotor abnormality and cognitive impairments in DCD for following reasons. First, the structural and functional integrity of striatum is essential for motor learning in subjects, a major defect occurred in DCD children, which promote us to make a speculation that affected striatum may partly underlie the mechanism of motor difficulties in DCD. Second, alterations in striatum are thought to contribute to impulsive behavior and hyperactivity through the inhibitive regulation. Thus, it is not difficult to understand that hyperactivity and impulsivity are frequently detected in DCD children with a comorbidity of ADHD $(15,44)$, all of which are related to hypofunction of striatum (30). Third, striatum deficiency early in development is thought to cause the nearly universal occurrence of compulsive behaviors in individuals. It is interestingly reported that DCD children have been shown to be at higher risk for obesity $(45,46)$, recently as a likely consequence of compulsive binge eating, which is related to disrupted striatal dopamine homeostasis. Collectively, with the increased awareness of striatal dual-directed action-cognition regulation, striatum is beginning to elucidate the mechanisms mediating both sensorimotor and cognition defects in DCD children.

Besides, evidence from animal studies may provide cues for the clinicians for discovering those once unidentified or unrecognized behavioral phenotypes in DCD children. For example, the ability to learn the temporal order of behavioral elements is central to the production of adaptive behavior including motor learning. Using a two-action sequence task without instructive cues in mice, Yin (47) found that excitotoxic lesions of the sensorimotor (dorsolateral) striatum dramatically impaired the acquisition of a simple sequence, implying the sensorimotor striatum necessary for serial-order learning. As DCD is featured as a sensorimotor disorder possibly due to striatum involvement, it could be speculated that a likely deficit in serial-order learning might be found in DCD children. However, whether this speculated symptom occurs in DCD and what mechanisms the striatum involves require further investigations.

In summary, accumulated studies indicate that multiple diffuse, rather than specific, brain areas may be involved in DCD children, which consequently bring them about a wide range of cognitive impairments (e.g., attention-deficit and/or language disorder) except sensorimotor difficulties. Further research into the affected brain areas at the neuronal circuit level, especially in conjunction with the relative symptoms, will help to better understand the etiology of DCD and refine intervention of the related sensorimotor-congnitive impairments.

\section{AFFECTED SYNAPTIC TRANSMISSION IMPLICATED IN DCD}

\section{Synaptic Plasticity and Neural Circuit Formation}

Recently, more and more animal studies on motor and cognitive activity at the synaptic level shed light on the mechanisms of sensorimotor impairment and cognition abnormality in DCD children. Changes in synaptic connections are long considered essential for learning and memory formation (48-53). Earlier studies showed that novel motor-skill learning increased the efficacy of synapses in the primary motor cortex (54-56). However, how motor learning affects neuronal circuitry at the level of individual synapses and how long-lasting memory is structurally encoded in the intact brain remain unknown. Xu et al. (57) reported that rapid, but long-lasting, synaptic reorganization was closely associated with motor learning, and stabilized neuronal connections were the foundation of durable motor memory. These results were confirmed 


\section{Mechanisms for DCD cognitive impairments}

by others that minute but permanent mark on cortical connections and lifelong memories were stored in largely stable, connected synaptic networks (58). For the abnormal cognition in DCD children, synapse connection and synaptic networks may change in the brain of these children.

As shown before, it is an increasingly accepted idea that sensorimotor activity modulates cognitive development. However, there was less experimental evidence supporting this idea at the synapse level. Animal experiments have shown that forced or voluntary physical activity such as wheel running promotes dentate gyrus neurogenesis in rodents, facilitates induction of long-term potentiation (LTP) underlying synaptic plasticity, and therefore enhances learning and memory performance $(59,60)$. To answer the question whether specific fine motor training can also augment synaptic plasticity and promote cognitive development, Zhang et al. (61) in our group performed the rodent forepaw sensorimotor deprivation in early development and consequently found impaired synaptic plasticity in hippocampal medial perforant-dentate gyrus pathway as well as disrupted spatial memory. On the other hand, sensorimotor training also leads to increased LTP as well as enhanced memory, just like abnormal sensorimotor deprivation causes depressive LTP and impaired memory. Recently, the work of Zhuang suggests that aberrant plasticity also contribute to abnormal motor control. He has demonstrated in his work that the aberrant plasticity at corticostriatal synapses caused by dopamine deficiency can result in disrupted motor learning, which specifically affect the learned motor skill rather than newly acquired motor skill. This finding may shed light on the potential mechanism underlying sever motor difficulties occurred in some diseases including DCD and Parkinson's disease (62). Taken together, the dysregulation of synaptic plasticity may account for both disrupted motor control and impaired cognition, indicating possible altered synapses function involved in DCD patients, which need further investigation.

\section{NEUROTRANSMITTER/RECEPTOR AND MOLECULAR MECHANISMS}

The well-established transmitter and receptor mechanism in other neurological diseases and related animal models may shed light on the studies about mechanism and intervention on young DCD patients. The prominent defects of motor learning in DCD argue the intact transmitter and receptors in the related brain regions, such as the major excitatory transmitter receptor-N-methyl-D-aspartate receptor (NMDAR) in the striatum. Corresponding to this speculation, a striatumspecific NMDAR1 subunit-knockout mice was generated and found that the abolishment of NMDAR activity in the striatum resulted in minor changes in striatal neuronal morphology, but dramatic disruption in dorsal striatal LTP and ventral striatal long-term depression, as well as severe impairment in motor learning (63). These findings from animal studies indicate that the similar disturbed striatal NMDAR might attribute to abnormality of motor learning in some human diseases, such as DCD in children and Parkinson's disease in the elderly. Furthermore, some molecular pathway, for example, the abnormal hippocampal cyclic adenosine monophosphate (cAMP) response element-binding signaling, may also associate to altered sensorimotor input and disturbed spatial memory. In our studies, we found that memory impairments displayed in early sensorimotor-deprived rats were associated with suppressed hippocampal neurogenesis and decreased cAMP response element-binding signaling, suggesting neurogenesis involvement in the sensorimotor-cognition interaction (64).

Some neurotransmitters and receptors for inhibition are also critical for sensorimotor-cognition interaction. When the major inhibitory $\gamma$-aminobutyric acid A (GABA) receptor that mediated synaptic inhibition was selectively removed from Purkinje cells, the consolidation of vestibulocerebellar motor learning could not be conducted successfully (65). Moreover, even removal of GABAA receptor $\gamma 2$ subunits from parvalbumin neurons expressing fast spiking caused wideranging behavioral alterations, such as severe impairments of motor skills and spatial learning (66). These results indicate that GABAergic inhibition in interneuron is indispensable for sensorimotor-cognition interaction, again suggesting that the abnormality of the above mechanisms probably confers clinical syndromes in DCD children.

Although clinical evidences suggest a genetic linkage between DCD and ADHD children $(67,68)$, the molecular mechanisms of DCD are still unclear. As the children with DCD often meet the criteria for ADHD, the molecular mechanisms in the latter partially involves the pathogenesis of DCD. ADHD may in part result from deficits in the dopaminergic system in cortical brain structures such as the PFC (69) and subcortical areas such as the nucleus accumbens and the striatum (70). Dopamine is particularly effective in modulating the activities of hyperexcitable young neurons (71), and neurogenesis is related with skilled forelimb training (72). Besides, dopaminergic D1/D5 receptor-mediated processes are important for certain forms of memory and its cellular model, i.e., hippocampal LTP in CA1. The synergistic role of D1/D5 as well as NMDA receptor function is required for the induction of the protein synthesis-dependent maintenance of CA1-LTP (late LTP) by activating the cAMP/PKA pathway (73). Therefore, the involvement of dopamine transporter and dopamine receptors, DRD4 and DRD5, in ADHD should be preferentially investigated in DCD children in the future. Except dopamine, other molecular candidates which are probably associated with dopamine function, such as $G$ protein-coupled receptor kinase-interacting protein-1 (74) and membrane receptor guanylyl cyclase-C (75), both implicated in ADHD, are encouraged to examine in DCD children in the future. Collectively, synaptic transmission and plasticity implicated in motor learning and other cognitive components probably interplay between sensorimotor and cognition in normal and DCD children, which needs to be proven in future.

\section{RECOVERY OF COGNITIVE IMPAIRMENT AND SENSORIMOTOR DYSFUNCTION IN DCD}

Clinical treatment approaches used for DCD (76-78) can be generally divided into two types: process or deficit-oriented 
and task-specific (14,78). A lot of the literature about interventions for DCD recently confirmed that interventions have been shown to produce benefits for the motor performance of DCD children. Moreover, approaches from a task-oriented perspective yield stronger effects (79). Currently, many studies about intervention focus more on the effectiveness evaluation of motor domain but less on that of cognition domain.

Given the extensive effect of sensorimotor regulation on cognition in normal and abnormal subjects even with DCD, the approaches for sensorimotor enhancement may also facilitate impaired cognition. In rats, task-specific motor enrichment procedures improved functional and neural outcomes following unilateral infusions of 6-hydroxydopamine into the nigrostriatal pathway (80). In addition, in focal cortical infarct animal model, skilled forelimb training effectively stimulated dentate neurogenesis in both infarcted and healthy brain and therefore enhanced spatial learning (72). In our previous study, we found that when the fine motor of young rats was deprived, hippocampal pyramidal cell numbers decreased, NMDAR1 expression altered, and subsequent learning and memory impaired (81). These might be the cellular mechanism for high efficacy of task-specific intervention on cognition. Altogether, it is likely that taskspecific intervention could also be useful for impaired cognition in DCD children. Moreover, further investigation on the cognitive effectiveness of current approaches need to be conducted on DCD children.

\section{SUMMARY AND PERSPECTIVES}

DCD is featured as a sensorimotor disability, accompanied by cognition abnormality. In this review, we discussed the possible systematic, circuit-level, synaptic, and transmitter/receptor mechanisms underlying the interactive sensorimotorcognition phenotypes. Based on the understanding of such mechanisms, a therapeutic strategy of cognition enhancement through sensorimotor manipulation was proposed, which will provide cues for the intervention and understanding of DCD in the future. Many human and animal studies remain to be performed regarding the structure and function of synaptic connection changes, including the levels of neurotransmitter, structure and function of receptors, and synapse plasticity. In addition, the molecular mechanism of DCD still needs to be confirmed. As of now, children with DCD, with appropriate training, are able to learn complex motor skills (82) but are not able to master them. The best intervention, based on neurological evidence, addressing both motor and cognition symptoms of DCD needs to be developed.

\section{STATEMENT OF FINANCIAL SUPPORT}

This study was supported by the Ministry of Education of China (NCET Program), National Science Foundation of China (81000592, 81222012, and 912321023), the National Basic Research Program of China (2013CB835100), Science and Technology Commission of Shanghai Municipality (10DZ2272200, 09DZ2200900, 10PJ1407500, 10PJ1403500, 10231203903, and 10JC1411200), Shanghai Municipal Education Commission (11ZZ103), Shanghai Municipal Health Bureau (2010004), Morning Star Rewarding Fund (Category B, 2011) of Shanghai Jiao Tong University, Xingbairen Plan of Shanghai Jiao Tong University, School of Medicine, internal fund of Shanghai
Children's Medical Center, and the Excellent Young College Teachers Fund of Shanghai Municipal Education Commission.

\section{REFERENCES}

1. American Psychiatric Association. Diagnostic and Statistical Manual of Mental Disorders, 4th edn. Washington, DC: American Psychiatric Publishing, Inc, 2000.

2. Zwicker JG, Missiuna C, Harris SR, Boyd LA. Developmental coordination disorder: a review and update. Eur J Paediatr Neurol 2012;16:573-81.

3. Geuze RH. Postural control in children with developmental coordination disorder. Neural Plast 2005;12:183-96; discussion 263-72.

4. Johnston LM, Burns YR, Brauer SG, Richardson CA. Differences in postural control and movement performance during goal directed reaching in children with developmental coordination disorder. Hum Mov Sci 2002;21:583-601.

5. Lundy-Ekman L, Ivry R, Keele S, Woollacott M. Timing and force control deficits in clumsy children. J Cogn Neurosci 1991;3:367-76.

6. Estil LB, Ingvaldsen RP, Whiting HT. Spatial and temporal constraints on performance in children with movement co-ordination problems. Exp Brain Res 2002;147:153-61.

7. Piek JP, Skinner RA. Timing and force control during a sequential tapping task in children with and without motor coordination problems. J Int Neuropsychol Soc 1999;5:320-9.

8. Henderson L, Rose P, Henderson S. Reaction time and movement time in children with a Developmental Coordination Disorder. J Child Psychol Psychiatry 1992;33:895-905.

9. Raynor AJ. Fractioned reflex and reaction time in children with developmental coordination disorder. Motor Control 1998;2:114-24.

10. Volman M, Geuze R. Relative phase stability of bimanual and visuomanual rhythmic coordination patterns in children with a developmental coordination disorder. Hum Mov Sci 1998;17:541-72.

11. Visser J. Developmental coordination disorder: a review of research on subtypes and comorbidities. Hum Mov Sci 2003;22:479-93.

12. Rivard L, Missiuna C, Pollock N, David KS. Developmental coordination disorder (DCD). In: Campbell SK, Palisano RJ, Orlin MN, eds. Physical Therapy for Children, 4th edn. St. Louis, MO: Elsevier, 2011:498-538.

13. Polatajko H. Developmental coordination disorder (DCD): alias the clumsy child syndrome. In: Whitmore K, Hart H, Willems G, eds. A Neurodevelopmental Approach to Specific Learning Disorders. Clinics in Developmental Medicine, no. 145. London, UK: MacKeith Press, 1999:119-33.

14. Polatajko HJ, Cantin N. Developmental coordination disorder (dyspraxia): an overview of the state of the art. Semin Pediatr Neurol 2005;12:250-8.

15. Kadesjö B, Gillberg C. Developmental coordination disorder in Swedish 7-year-old children. J Am Acad Child Adolesc Psychiatry 1999;38:820-8.

16. Dewey D, Kaplan BJ, Crawford SG, Wilson BN. Developmental coordination disorder: associated problems in attention, learning, and psychosocial adjustment. Hum Mov Sci 2002;21:905-18.

17. Kadesjö B, Gillberg C. Attention deficits and clumsiness in Swedish 7-yearold children. Dev Med Child Neurol 1998;40:796-804.

18. Piek JP, Dyck MJ. Sensory-motor deficits in children with developmental coordination disorder, attention deficit hyperactivity disorder and autistic disorder. Hum Mov Sci 2004;23:475-88.

19. Piek JP, Dyck MJ, Nieman A, et al. The relationship between motor coordination, executive functioning and attention in school aged children. Arch Clin Neuropsychol 2004;19:1063-76.

20. Watemberg N, Waiserberg N, Zuk L, Lerman-Sagie T. Developmental coordination disorder in children with attention-deficit-hyperactivity disorder and physical therapy intervention. Dev Med Child Neurol 2007;49:920-5.

21. Archibald LM, Alloway TP. Comparing language profiles: children with specific language impairment and developmental coordination disorder. Int J Lang Commun Disord 2008;43:165-80.

22. Alloway TP. The neurophysiological performance of visuospatial working memory in children with developmental coordination disorder. Dev Med Child Neurol 2012;54:1075-6.

23. Clements SD, Peters JE. Minimal brain dysfunctions in the school-age child. Diagnosis and treatment. Arch Gen Psychiatry 1962;6:185-97. 


\section{Mechanisms for DCD cognitive impairments $\quad$ RevieW}

24. Gilger JW, Kaplan BJ. Atypical brain development: a conceptual framework for understanding developmental learning disabilities. Dev Neuropsychol 2001;20:465-81.

25. Zwicker JG, Missiuna C, Harris SR, Boyd LA. Brain activation associated with motor skill practice in children with developmental coordination disorder: an fMRI study. Int J Dev Neurosci 2011;29:145-52.

26. Krakauer JW, Shadmehr R. Towards a computational neuropsychology of action. Prog Brain Res 2007;165:383-94.

27. Kawato M. Internal models for motor control and trajectory planning. Curr Opin Neurobiol 1999;9:718-27.

28. Passot JB, Sheynikhovich D, Duvelle É, Arleo A. Contribution of cerebellar sensorimotor adaptation to hippocampal spatial memory. PLoS ONE 2012;7:e32560.

29. Zwicker JG, Missiuna C, Harris SR, Boyd LA. Brain activation of children with developmental coordination disorder is different than peers. Pediatrics 2010;126:e678-86.

30. Querne L, Berquin P, Vernier-Hauvette MP, et al. Dysfunction of the attentional brain network in children with Developmental Coordination Disorder: a fMRI study. Brain Res 2008;1244:89-102.

31. Yang Y, Raine A. Prefrontal structural and functional brain imaging findings in antisocial, violent, and psychopathic individuals: a meta-analysis. Psychiatry Res 2009;174:81-8.

32. Miller EK, Freedman DJ, Wallis JD. The prefrontal cortex: categories, concepts and cognition. Philos Trans R Soc Lond, B, Biol Sci 2002;357:1123-36.

33. Robbins TW. Dissociating executive functions of the prefrontal cortex. Philos Trans R Soc Lond, B, Biol Sci 1996;351:1463-70; discussion 1470-1.

34. Alvarez JA, Emory E. Executive function and the frontal lobes: a metaanalytic review. Neuropsychol Rev 2006;16:17-42.

35. Goldman-Rakic PS. Topography of cognition: parallel distributed networks in primate association cortex. Annu Rev Neurosci 1988;11:137-56.

36. Price JL. Prefrontal cortical networks related to visceral function and mood. Ann N Y Acad Sci 1999;877:383-96.

37. Cantell M, Smyth M, Ahonen T. Clumsiness in adolescence: educational, motor, and social outcomes of motor delay detected at 5 years. Adapt Phys Activ Q 1994;11:115-29.

38. Bouffard M, Watkinson E, Thompson L, Causgrove-Dunn J. A test of the activity deficit hypothesis with children with movement difficulties. Adapt Phys Activ Q 1996;13:61-73.

39. Chen HF, Cohn ES. Social participation for children with developmental coordination disorder: conceptual, evaluation and intervention considerations. Phys Occup Ther Pediatr 2003;23:61-78.

40. Missiuna C, Moll S, King S, King G, Law M. A trajectory of troubles: parents' impressions of the impact of developmental coordination disorder. Phys Occup Ther Pediatr 2007;27:81-101.

41. Missiuna C, Moll S, King G, Stewart D, Macdonald K. Life experiences of young adults who have coordination difficulties. Can J Occup Ther 2008;75:157-66.

42. Van den Bercken JH, Cools AR. Evidence for a role of the caudate nucleus in the sequential organization of behavior. Behav Brain Res 1982;4:319-27.

43. Voytek B, Knight RT. Prefrontal cortex and basal ganglia contributions to visual working memory. Proc Natl Acad Sci USA 2010;107:18167-72.

44. Missiuna C, Cairney J, Pollock N, Cousins M, MacDonald K. Exploring the Psychological Consequences of Developmental Coordination Disorder. Can Assoc Occup Ther Conf Proc. Ottawa, CA: CAOT; 2009.

45. Cairney J, Hay JA, Faught BE, Hawes R. Developmental coordination disorder and overweight and obesity in children aged 9-14 y. Int J Obes (Lond) 2005;29:369-72.

46. Cairney J, Hay J, Veldhuizen S, Missiuna C, Mahlberg N, Faught BE. Trajectories of relative weight and waist circumference among children with and without developmental coordination disorder. CMAJ 2010;182:116772.

47. Yin $\mathrm{HH}$. The sensorimotor striatum is necessary for serial order learning. J Neurosci 2010;30:14719-23.

48. Bailey CH, Kandel ER. Structural changes accompanying memory storage. Annu Rev Physiol 1993;55:397-426.

49. Buonomano DV, Merzenich MM. Cortical plasticity: from synapses to maps. Annu Rev Neurosci 1998;21:149-86.
50. Changeux JP, Danchin A. Selective stabilisation of developing synapses as a mechanism for the specification of neuronal networks. Nature 1976;264:705-12.

51. Hubel DH, Wiesel TN, LeVay S. Plasticity of ocular dominance columns in monkey striate cortex. Philos Trans R Soc Lond, B, Biol Sci 1977;278:377409.

52. Lichtman JW, Colman H. Synapse elimination and indelible memory. Neuron 2000;25:269-78.

53. Shatz CJ, Stryker MP. Ocular dominance in layer IV of the cat's visual cortex and the effects of monocular deprivation. J Physiol (Lond) 1978;281:26783.

54. Rioult-Pedotti MS, Donoghue JP, Dunaevsky A. Plasticity of the synaptic modification range. J Neurophysiol 2007;98:3688-95.

55. Rioult-Pedotti MS, Friedman D, Donoghue JP. Learning-induced LTP in neocortex. Science 2000;290:533-6.

56. Harms KJ, Rioult-Pedotti MS, Carter DR, Dunaevsky A. Transient spine expansion and learning-induced plasticity in layer 1 primary motor cortex. J Neurosci 2008;28:5686-90.

57. Xu T, Yu X, Perlik AJ, et al. Rapid formation and selective stabilization of synapses for enduring motor memories. Nature 2009;462:915-9.

58. Yang G, Pan F, Gan WB. Stably maintained dendritic spines are associated with lifelong memories. Nature 2009;462:920-4.

59. van Praag H, Christie BR, Sejnowski TJ, Gage FH. Running enhances neurogenesis, learning, and long-term potentiation in mice. Proc Natl Acad Sci USA 1999;96:13427-31.

60. Farmer J, Zhao X, van Praag H, Wodtke K, Gage FH, Christie BR. Effects of voluntary exercise on synaptic plasticity and gene expression in the dentate gyrus of adult male Sprague-Dawley rats in vivo. Neuroscience 2004;124:71-9.

61. Zhang Y, Li F, Cao X, et al. Forepaw sensorimotor deprivation in early life leads to the impairments on spatial memory and synaptic plasticity in rats. J Biomed Biotechnol 2009;2009:919276.

62. Zhuang XX. Aberrant plasticity and "learned" motor inhibition in Parkinson's disease. Sheng Li Xue Bao 2012;64:543-9.

63. Dang MT, Yokoi F, Yin HH, Lovinger DM, Wang Y, Li Y. Disrupted motor learning and long-term synaptic plasticity in mice lacking NMDAR1 in the striatum. Proc Natl Acad Sci USA 2006;103:15254-9.

64. Li F, Zhang YY, Jing XM, Yan CH, Shen XM. Memory impairment in early sensorimotor deprived rats is associated with suppressed hippocampal neurogenesis and altered CREB signaling. Behav Brain Res 2010;207:458-65.

65. Wulff P, Schonewille M, Renzi M, et al. Synaptic inhibition of Purkinje cells mediates consolidation of vestibulo-cerebellar motor learning. Nat Neurosci 2009;12:1042-9.

66. Leppä E, Linden AM, Vekovischeva OY, et al. Removal of GABA(A) receptor ?2 subunits from parvalbumin neurons causes wide-ranging behavioral alterations. PLoS ONE 2011;6:e24159.

67. Martin NC, Piek JP, Hay D. DCD and ADHD: a genetic study of their shared aetiology. Hum Mov Sci 2006;25:110-24.

68. Fliers E, Vermeulen S, Rijsdijk F, et al. ADHD and poor motor performance from a family genetic perspective. J Am Acad Child Adolesc Psychiatry 2009;48:25-34.

69. Sullivan RM, Brake WG. What the rodent prefrontal cortex can teach us about attention-deficit/hyperactivity disorder: the critical role of early developmental events on prefrontal function. Behav Brain Res 2003;146:43-55.

70. Russell V, de Villiers A, Sagvolden T, Lamm M, Taljaard J. Altered dopaminergic function in the prefrontal cortex, nucleus accumbens and caudateputamen of an animal model of attention-deficit hyperactivity disorderthe spontaneously hypertensive rat. Brain Res 1995;676:343-51.

71. Mu Y, Zhao C, Gage FH. Dopaminergic modulation of cortical inputs during maturation of adult-born dentate granule cells. J Neurosci 2011;31:4113-23.

72. Wurm F, Keiner S, Kunze A, Witte OW, Redecker C. Effects of skilled forelimb training on hippocampal neurogenesis and spatial learning after focal cortical infarcts in the adult rat brain. Stroke 2007;38:2833-40.

73. Navakkode S, Sajikumar S, Frey JU. Synergistic requirements for the induction of dopaminergic D1/D5-receptor-mediated LTP in hippocampal slices of rat CA1 in vitro. Neuropharmacology 2007;52:1547-54. 
74. Won H, Mah W, Kim E, et al. GIT1 is associated with ADHD in humans and ADHD-like behaviors in mice. Nat Med 2011;17:566-72.

75. Gong R, Ding C, Hu J, et al. Role for the membrane receptor guanylyl cyclase-C in attention deficiency and hyperactive behavior. Science 2011;333:1642-6.

76. Mandich AD, Polatajko HJ, Macnab JJ, Miller LT. Treatment of children with Developmental Coordination Disorder: what is the evidence? Phys Occup Ther Pediatr 2001;20:51-68.

77. Wilson PH. Practitioner review: approaches to assessment and treatment of children with DCD: an evaluative review. J Child Psychol Psychiatry 2005;46:806-23.

78. Sugden D. Current approaches to intervention in children with developmental coordination disorder. Dev Med Child Neurol 2007;49:467-71.
79. Smits-Engelsman BC, Blank R, van der Kaay AC, et al. Efficacy of interventions to improve motor performance in children with developmental coordination disorder: a combined systematic review and meta-analysis. Dev Med Child Neurol 2013;55:229-37.

80. Ciucci MR, Ma ST, Kane JR, Ahrens AM, Schallert T. Limb use and complex ultrasonic vocalization in a rat model of Parkinson's disease: deficittargeted training. Parkinsonism Relat Disord 2008;14:Suppl 2:S172-5.

81. Li F, Zhang YY, Jing XM, Yan CH, Shen XM. The influence of forepaw palmar sensorimotor deprivation on learning and memory in young rats. Neurosci Res 2009;63:17-23.

82. Snapp-Childs W, Mon-Williams M, Bingham GP. A sensorimotor approach to the training of manual actions in children with developmental coordination disorder. J Child Neurol 2013;28:204-12. 